\title{
High-flow nasal cannula support therapy: new insights and improving performance
}

\author{
Gonzalo Hernández ${ }^{1 *}$, Oriol Roca ${ }^{2,3}$ and Laura Colinas ${ }^{1}$
}

\begin{abstract}
This article is one of ten reviews selected from the Annual Update in Intensive Care and Emergency Medicine 2017. Other selected articles can be found online at http://ccforum.com/series/annualupdate2017. Further information about the Annual Update in Intensive Care and Emergency Medicine is available from http://www.springer.com/series/8901.
\end{abstract}

\section{Background}

Oxygen therapy is the first step in the prevention and treatment of hypoxemic respiratory failure and has traditionally been delivered using nasal prongs or masks. However, the maximal flow rates that these devices can deliver are limited because of the discomfort generated secondary to insufficient heat and humidity provided to the gas administered. Although high-flow oxygen therapy is currently defined as flows greater than $30 \mathrm{l} / \mathrm{min}$, it is accepted that flows up to $15 \mathrm{l} / \mathrm{min}$ can be delivered using conventional nasal prongs or masks; this flow is far less than the peak inspiratory flow of a patient with dyspnea. In addition, flows exceeding $6 \mathrm{l} / \mathrm{min}$ can lead to insufficient humidification provided to the nasal mucosa, even when a cold bubble humidifier is used. Therefore, room air dilutes the supplemental oxygen, resulting in a significant decrease in the fraction of the inspired oxygen $\left(\mathrm{FiO}_{2}\right)$ that finally reaches the alveoli.

In recent years, new devices that deliver totally conditioned gas $\left(37{ }^{\circ} \mathrm{C}\right.$ containing $44 \mathrm{mg} \mathrm{H}_{2} \mathrm{O} / 1[100 \%$ relative humidity] using a heated humidifier and a heated inspiratory circuit) through a wide bore nasal cannula at very high flow (up to $60 \mathrm{l} / \mathrm{min}$ ) at a predetermined constant oxygen concentration (21 to 100\%) have emerged as a safe and useful supportive therapy in many clinical situations.

\footnotetext{
* Correspondence: ghernandezm@telefonica.net

${ }^{1}$ Department of Critical Care Medicine, Virgen de la Salud University Hospital, Ave de Berber, 45005 Toledo, Spain

Full list of author information is available at the end of the article
}

High-flow nasal cannula (HFNC) supportive therapy exerts its potential benefits through a variety of mechanisms. In the literature this treatment strategy has been variously described as nasal high flow and high flow oxygen therapy, but we believe that the most appropriate term is heated and humidified HFNC supportive therapy. This term reflects the features that generate the technique's clinical effects (i. e., the delivery of warm and humidified air at high flows through a nasal cannula).

\section{New insights \\ Physiological effects and consequences \\ Heated and humidified oxygen}

Inhaling dry and cold oxygen provokes upper airway dryness frequently leading to intolerance, and potentially impairing mucociliary functions, such as secretion clearance and airway defense. Results of studies demonstrate that HFNC reduces patient discomfort and upper airway dryness, although a potentially protective effect on mucociliary function requires further investigation. Another way in which HFNC improves extubation outcome and weaning is by conditioning the inspired gas. Various studies have demonstrated that HFNC improves the management of respiratory secretions and reported fewer reintubations secondary to upper airway obstruction and accelerated weaning in tracheostomy patients. These findings support the idea that gas conditioning probably alleviates inflammation of the tracheal mucosa after transglottic intubation, and a protocol that includes the use of HFNC prior to extubation, thereby preventing the administration of dry and cold air in the native airway of the patients, reinforces this approach [1].

\section{Carbon dioxide clearance}

There is still not much information about the role of HFNC in managing hypercapnia, except for the mechanism of dead-space washout. By providing a high flow of fresh air during expiration, HFNC may be able to more rapidly washout the carbon dioxide 
$\left(\mathrm{CO}_{2}\right)$ filling the nasopharyngeal cavity. Möller et al. constructed an airway model using a computed tomography (CT) scan, and analyzed the lavage of gas tracers under apneic conditions. The authors observed a linear positive correlation between tracer-gas clearance in the model and the flow rate of HFNC, approximately $1.8 \mathrm{ml} / \mathrm{s}$ increase in clearance for every $1.0 \mathrm{l} / \mathrm{min}$ increase in flow [2].

However, in recent years, new studies have highlighted some additional mechanisms affecting $\mathrm{CO}_{2}$ clearance. Alveolar ventilation has been suggested by Patel et al., after obtaining a mean apnea time of $17 \mathrm{~min}$ in surgical patients [3]. The authors reported further evidence that classical apneic oxygenation provided little clearance of $\mathrm{CO}_{2}$ apart from that obtained from limiting rebreathing. Continuous insufflation of a high-flow gas mixture facilitates oxygenation and $\mathrm{CO}_{2}$ clearance through gaseous mixing. Evidence for the existence of flow-dependent, non-rhythmic ventilatory exchange can be provided by comparing the increase in rise of $\mathrm{CO}_{2}$ under different continuous insufflation apneic conditions. While Rudolf and Hohenhorst [4] reported a rate of carbon dioxide increase of $0.24 \mathrm{kPa} / \mathrm{min}$ using a low-flow oxygen intratracheal cannula $(0.5 \mathrm{l} / \mathrm{min})$, Patel et al. [3] achieved a rate of carbon dioxide increase of $0.15 \mathrm{kPa} / \mathrm{min}$ and a steady-state carbon dioxide level was not reached. This result was improved only when using a high-flow oxygen intratracheal cannula $(45 \mathrm{l} / \mathrm{min})$, reaching a steady-state carbon dioxide level within $5 \mathrm{~min}$ of the start of the apnea [4].

Recently, Hernandez et al. [5] reported data supporting a possible role of HFNC in managing hypercapnia after extubation. The investigators compared non-invasive ventilation (NIV) to HFNC in a fixed $24 \mathrm{~h}$ protocol after extubation in high-risk for reintubation patients, using a non-inferiority randomized trial. There was a trend towards a higher rates of postextubation respiratory failure due to hypercapnia in the NIV group than in the HFNC group, although this difference was not translated to the rates of hypercapnia as the reason for reintubation. They argued that the real time under NIV, clearly inferior to the $24 \mathrm{~h}$ expected by protocol, suggests that discomfort could have been the reason for hypercapnic postextubation respiratory failure in some NIV patients, as $\mathrm{PaCO}_{2}$ improved without any respiratory support in most patients. The reported rates of reintubation due to hypercapnia were similar in the two groups, as were the values of gasometric variables at reintubation, suggesting that high-flow is at least as good as NIV for managing postextubation hypercapnia in high-risk patients.

\section{Positive pharyngeal pressure}

HFNC can induce a positive pharyngeal pressure during expiration due to its constant ingoing flow, with the effect mainly determined by the flow rate provided by HFNC and the expiratory flow exhaled by the patient, with lower pressures when the patient keeps the mouth open.

Parke and McGuinness [6] reported that HFNC increased the mean pharyngeal pressure by about 1 $\mathrm{cmH}_{2} \mathrm{O}$ per $10 \mathrm{l} / \mathrm{min}$, within a range of $30-50 \mathrm{l} / \mathrm{min}$, but more recently [7], the same group reported that the linear increase in the pharyngeal pressure was maintained when using extra high-flow with $100 \mathrm{l} / \mathrm{min}$ (combining two HFNC systems), obtaining pharyngeal pressures up to $11.9 \mathrm{cmH}_{2} \mathrm{O}$. However, these values were obtained in healthy volunteers and extrapolation to the critical care arena may not be accurate.

Despite this uncertainty about how much positive endexpiratory pressure (PEEP) can really be offered by HFNC, studies [8] have demonstrated that end-expiratory lung impedance increases with rising flow rate of HFNC, suggesting an increase in end-expiratory lung volume. In addition, hemodynamic changes similar to those obtained in patients under NIV with pressure levels close to $10 \mathrm{cmH}_{2} \mathrm{O}$ have been reported [9]. Right atrial pressure has been considered as a surrogate of right ventricular preload and is most commonly estimated by inferior vena cava (IVC) diameter and the presence of inspiratory collapse. Changes in IVC diameter have been used to determine preload responsiveness in positive pressure ventilated patients. In a population of patients with New York Heart Association (NYHA) class III heart failure, treatment with HFNC at 20 and $40 \mathrm{l} / \mathrm{min}$ was associated with mean attributable reductions in the IVC inspiratory collapse of 20 and 53\% from baseline, respectively. These changes were reversible after HFNC withdrawal suggesting that HFNC therapy may modify the hemodynamic status in heart failure patients at the same rate as does NIV.

HFNC is, therefore, not entirely similar to applying continuous positive airway pressure (CPAP), which aims to maintain a steady level of positive pressure during the whole cycle of breath. The target of HFNC is flow instead of pressure, so the objective when applying HFNC should not be the pharyngeal pressure measured but the changes in hemodynamic status and the increase in lung aeration.

\section{Clinical data}

\section{Acute hypoxemic respiratory failure}

\section{Effects of HFNC on physiological variables and comfort}

The first studies in patients with acute respiratory failure focused on the effects of HFNC therapy on physiological variables [10-12] and reported oxygenation enhancement with reductions in respiratory rate and no changes in $\mathrm{PaCO}_{2}$ (Table 1). Moreover, HFNC appeared to be 


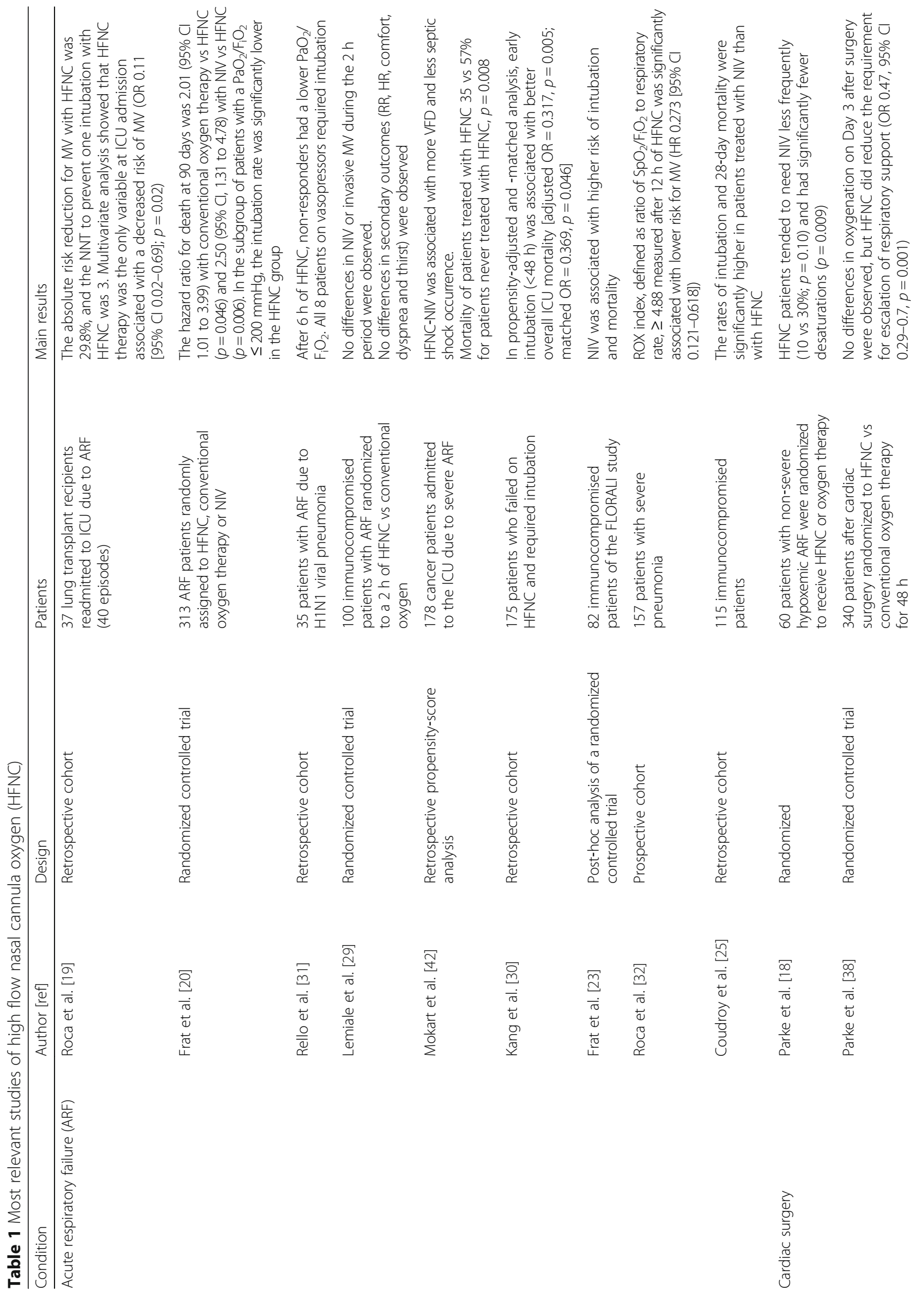




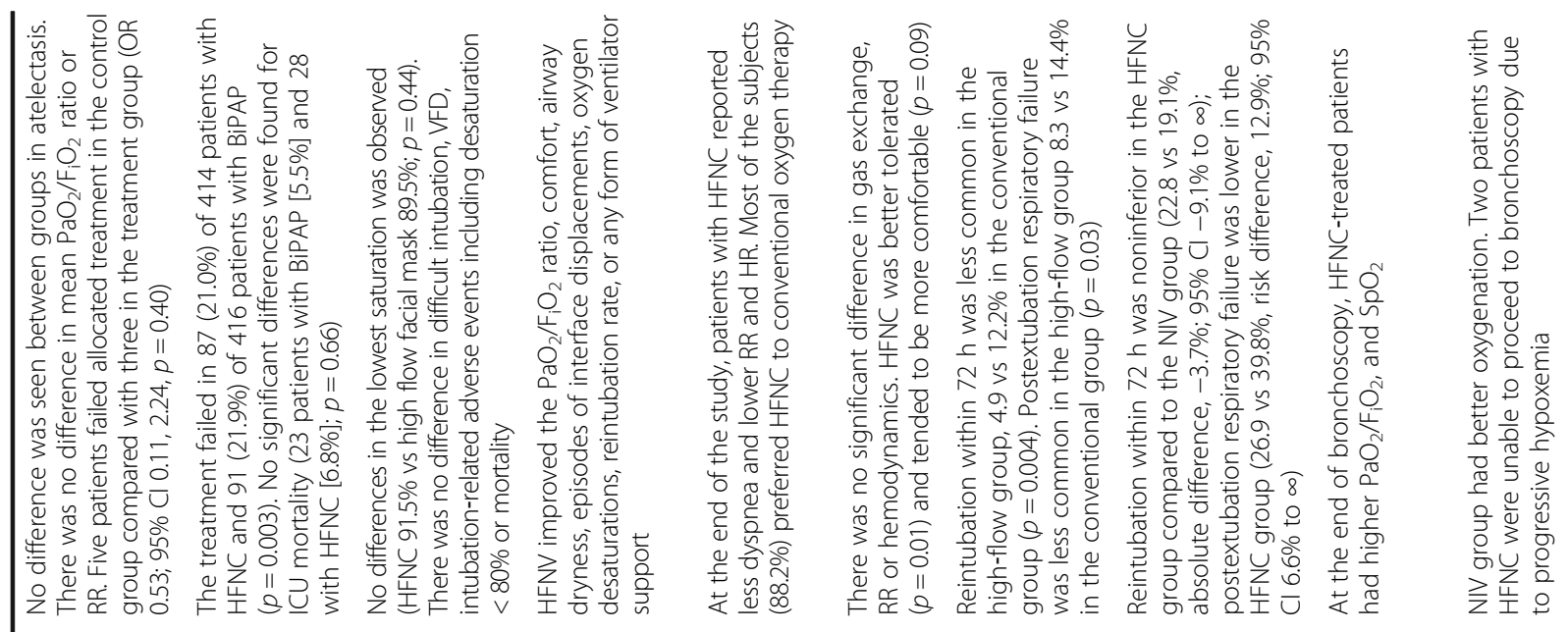

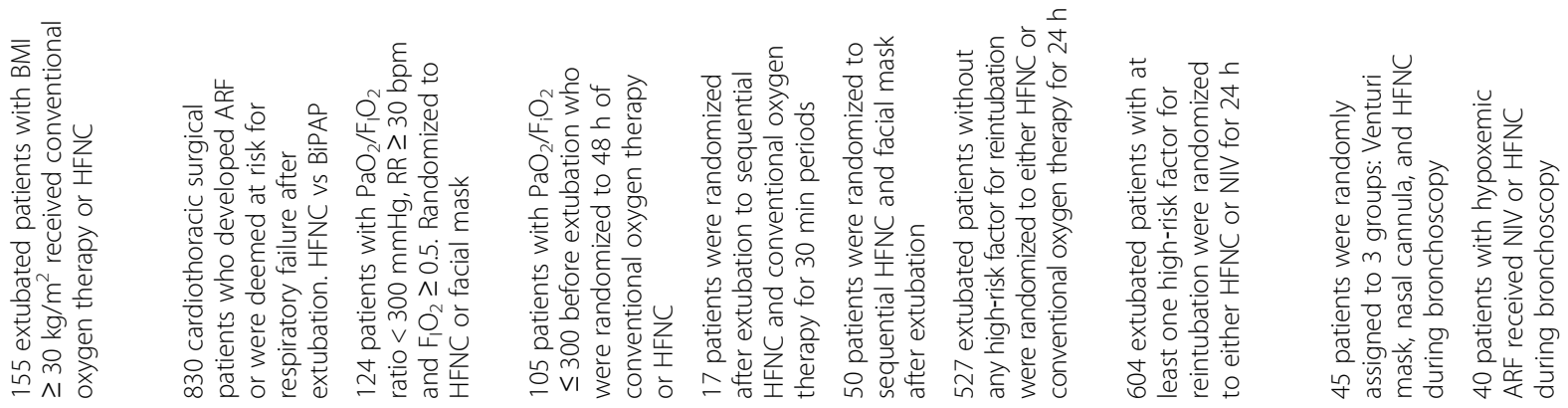


Hernández et al. Critical Care (2017) 21:62

Page 5 of 11

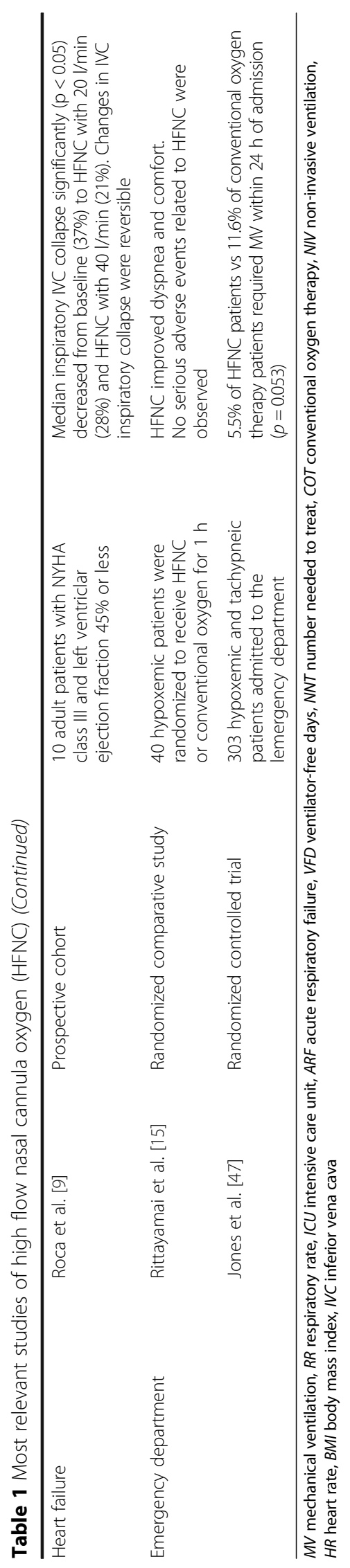


better tolerated and achieved a greater level of comfort than conventional oxygen devices [10, 12]. HFNC was also usually better tolerated than NIV, although NIV obtained greater improvements in oxygenation [13, 14].

In addition, HFNC has been used in small cohorts of patients with acute respiratory failure in the emergency department $[15,16]$ and even in 150 children less than 2 years old during interhospital transport [17]. The effects on oxygenation and rates of intubation reported in all these studies are similar to those found when HFNC has been used in patients with acute respiratory failure admitted to critical care areas, suggesting that, with adequate monitoring, HFNC can be safely used outside the critical care setting.

\section{Effects on intubation rate and mortality}

These physiological studies provide the rationale for considering HFNC as a potentially useful tool for decreasing intubation rates and mortality in patients with acute respiratory failure. The first randomized controlled trial (RCT) included postoperative cardiac surgery patients with mild to moderate acute respiratory failure [18]. In that preliminary trial, HFNC patients were less likely to need escalation to NIV and had fewer desaturations than those treated using a standard humidified high flow face mask. In another retrospective analysis of a prospectively assessed cohort of 37 lung transplant patients readmitted to the ICU because of acute respiratory failure, HFNC therapy was the only variable at ICU admission associated with a decreased risk of mechanical ventilation in the multivariate analysis [19]. The absolute risk reduction for mechanical ventilation with HFNC was $29.8 \%$, and only three patients needed to be treated with HFNC to prevent one intubation. Moreover, nonventilated patients had an increased survival rate.

More recently, the results of the first large RCT to assess clinical outcomes with HFNC (50 l/min), conventional oxygen devices and NIV have been published [20]. The study included 310 patients with de novo hypoxemic acute respiratory failure, defined as $\mathrm{PaO}_{2} / \mathrm{FiO}_{2}$ ratio $\leq 300$ $\mathrm{mmHg}$ or respiratory rate $>25 \mathrm{bpm}$ with $10 \mathrm{lpm}$ of $\mathrm{O}_{2}$. Patients with a history of chronic respiratory disease, including chronic obstructive pulmonary disease (COPD), as well as patients with acute cardiogenic pulmonary edema, severe neutropenia and hypercapnia $\left(\mathrm{PaCO}_{2}>45\right.$ $\mathrm{mmHg}$ ) were excluded, as were patients with other organ failures, including hemodynamic instability or vasopressors at the time of inclusion. The most frequent cause of acute respiratory failure was pneumonia $(75 \%)$ and $80 \%$ of the enrolled patients showed bilateral pulmonary infiltrates at study inclusion. The primary outcome (the rate of endotracheal intubation) did not differ significantly between the groups (HFNC $38 \%$ vs. standard oxygen $47 \%$ and NIV $50 \% ; p=0.18$ ).
This negative result may be due to the fact that the observed rate of intubation with standard oxygen was lower than expected and, therefore, the study may have been underpowered. However, a post hoc adjusted analysis including the 238 patients with a $\mathrm{PaO}_{2} / \mathrm{FiO}_{2}$ ratio $\leq$ $200 \mathrm{mmHg}$ found that HFNC reduced intubation rates $(p=0.009)$. In the entire cohort, HFNC increased ventilator-free days, reduced 90-day mortality and was associated with better comfort and lower dyspnea severity. In contrast, NIV patients had higher 90-day mortality rates than HFNC patients.

Mechanisms that may explain these findings are the small amount of positive airway pressure and increased $\mathrm{CO}_{2}$ excretion with HFNC, the overall reduction in the need for intubation and the lower incidence of septic shock. Moreover, important concerns have been raised regarding how NIV was applied. First, half of the patients in the NIV group were treated with NIV for less than eight hours during the first two days of randomization and received HFNC between NIV sessions. Second, high tidal volumes $(9.2 \pm 3.0 \mathrm{ml} / \mathrm{kg})$ were used, which may have aggravated the preexisting lung injury. In fact, patients with acute respiratory failure usually have high respiratory rates and minute volumes and are more likely to present patient-ventilator asynchronies. Third, relatively low levels of PEEP were used in potentially recruitable patients $(5 \pm 1$ $\mathrm{cmH}_{2} \mathrm{O}$ ). Fourth, intermittent sessions of NIV may induce the phenomenon of recruitment/derecruitment, generating ventilator-induced lung injury (VILI). Finally, NIV was delivered through a face mask although it has later been shown that a helmet interface may be associated with better outcomes [21, 22]. Thus, the NIV protocol in this study could be assumed to be close to what is real practice at the bedside in many centers treating acute respiratory failure with NIV, so an underestimation of the NIV treatment effect in a pragmatic trial cannot be ruled out.

More recently, a post hoc subgroup analysis in a subset of immunocompromised patients was performed on the FLORALI study [23]. Thirty patients treated with standard oxygen therapy, 26 treated with HFNC, and 26 treated with NIV were included. The intubation and mortality rates were significantly higher in patients randomly assigned to NIV and, apart from age, the use of NIV as the first-line therapy was the only variable independently associated with a higher risk of endotracheal intubation and mortality in the multivariate logistic regression analysis. Moreover, the expired tidal volume measured $1 \mathrm{~h}$ after NIV initiation was higher in the patients who died than in survivors $(11.1 \pm 2.6 \mathrm{ml} / \mathrm{kg}$ of predicted weight versus $7.6 \pm 3.1 \mathrm{ml} / \mathrm{kg} ; p=0.02)$. Similarly, higher tidal volumes have been associated with NIV failure [24]. 
Thus, the high tidal volumes and high transpulmonary pressures obtained during NIV may induce VILI in a pre-injured lung and may be at least partially responsible for NIV failure and higher mortality. These results were similar to those observed in a recently published 8-year observational study of a cohort of 115 immunocompromised patients [25].

Two recent systematic reviews and meta-analyses have also been published that evaluated the effectiveness of HFNC [26, 27]. In the first study, no differences in terms of mortality or higher respiratory support requirement was observed [26]. Most recently, Monro-Somerville et al. [27] found that, although HFNC appears to be well tolerated, no difference in intubation rates or mortality was observed in patients treated with HFNC compared to those treated with usual care (conventional oxygen or NIV). However, the required information size was not reached: in fact, only 30.6 and $62.3 \%$ of the estimated needed patients were included for the primary (mortality) and secondary (intubation) outcomes, respectively. Moreover, HFNC was shown to have better survival and lower intubation rates than conventional oxygen and, although no differences were observed compared with NIV, HFNC was better tolerated. Finally, certain methodological issues regarding some of the studies included in both meta-analyses should be noted. First, a study by Stephan et al. [28] was a positive non-inferiority trial comparing HFNC and NIV; however, in the meta-analysis it appears as a negative trial as no differences were observed between HFNC and NIV. Second, in a study by Lemiale et al. [29], HFNC was only used for a 2 htrial and in such a short period of time it would be difficult to observe any difference between treatments. Finally, and most importantly, studies included in both meta-analyses had very heterogeneous populations of acute respiratory failure patients in terms of severity with huge differences in risks of intubation.

\section{Predictors of HFNC success}

The existence of accurate, early predictors of HFNC success is important. Indeed, a recent propensity-score analysis associated early intubation (within the first $48 \mathrm{~h}$ ) with better ICU survival [30]. In spite of its limitations, the study by Kang et al. [30] raises an important issue the fact that delayed intubation may worsen the prognosis of patients treated with HFNC. Therefore, the ability to describe accurate predictors of HFNC success that can allow timely endotracheal intubation in patients who are likely to fail is a point of special interest. Sztrymf et al. [12] reported that respiratory rate as well as the percentage of patients exhibiting thoraco-abdominal asynchrony as early as 30 and 15 min after the beginning of HFNC were significantly higher in patients who required endotracheal intubation. Moreover, the $\mathrm{PaO}_{2} /$
$\mathrm{FiO}_{2}$ ratio $1 \mathrm{~h}$ after the start of $\mathrm{HFNC}$ was significantly lower in patients requiring invasive mechanical ventilation. Similarly, in a series of 20 patients with $\mathrm{H}_{1} \mathrm{~N}_{1}$ infection treated with $\mathrm{HFNC}$, worse $\mathrm{PaO}_{2} / \mathrm{FiO}_{2}$ ratios were observed in patients who required intubation after six hours of treatment [31]. Interestingly, a recent prospective study showed that patients with severe pneumonia who had a ROX index (defined as the ratio of $\mathrm{SpO}_{2} / \mathrm{FiO}_{2}$ to respiratory rate) $\geq 4.88$ after $12 \mathrm{~h}$ of HFNC therapy were less likely to be intubated, even after adjusting for potential covariates [32]. Moreover, among patients who were still on HFNC after $18 \mathrm{~h}$, the median change in the ROX index between 18 and $12 \mathrm{~h}$ was significantly higher in patients who did not require intubation. However, non-pulmonary severity has also been described as a good predictor of HFNC failure. Indeed, the presence of shock has been associated with a higher risk of mechanical ventilation [19, 23].

In a preliminary study by Hernandez et al. [33] titrating high-flow according to patient tolerance, a gas flow > $35 \mathrm{l} / \mathrm{min} 12 \mathrm{~h}$ after extubation predicted reintubation, suggesting that the flow tolerated by patients is a marker of severity; unfortunately, this result was not reproduced in the subsequent randomized trial [5].

\section{ARDS patients}

Another controversial issue is whether patients with bilateral infiltrates treated with HFNC can be considered as having acute respiratory distress syndrome (ARDS). In fact, most patients included in studies have bilateral infiltrates [20,32]. The Berlin definition of ARDS [34] requires a minimum of $5 \mathrm{cmH}_{2} \mathrm{O}$ of PEEP, and it has been shown that HFNC can provide a level of PEEP that is higher at peak expiratory pressure [7]. Moreover, ARDS does not begin at the time of mechanical ventilation onset. Therefore, it can be accepted that patients with a risk factor for ARDS, who are hypoxemic $\left(\mathrm{PaO}_{2} / \mathrm{FiO}_{2}\right.$ ratio $\left.\leq 300 \mathrm{mmHg}\right)$ and have bilateral infiltrates not fully explained by cardiac failure or fluid overload, may be considered as ARDS patients. In these patients, HFNC may achieve success rates similar to those of NIV [35].

\section{Prevention of postextubation respiratory failure and reintubation}

Extubation failure is an independent predictor of mortality. The development of therapies to prevent this has been focused on specific causes of reintubation (corticoids for larynx edema and NIV for hypercapnic respiratory failure in patients with chronic pulmonary diseases) and in patients with risk factors associated with extubation failure. Usually, extubated patients receive conventional oxygen therapy for correcting the oxygenation 
impairment. This system provides low flow and does not guarantee the $\mathrm{FiO}_{2}$.

\section{Low-risk-of-reintubation patients}

Two preliminary physiological studies comparing HFNC with conventional oxygen devices using a crossover design and during a short period of time after extubation have confirmed the consistent benefit of HFNC in terms of overall comfort. Rittayamai et al. [15] observed a decrease in respiratory rate and heart rate when comparing HFNC therapy at $35 \mathrm{l} / \mathrm{min}$ vs conventional oxygen devices at $6-10 \mathrm{l} / \mathrm{min}$ in 17 patients during a $30 \mathrm{~min}$ period. In contrast, Tiruvoipati et al. [36] found no change in these physiological variables when comparing $30 \mathrm{l} / \mathrm{min}$ delivered through HFNC or $15 \mathrm{l} / \mathrm{min}$ through a high flow face mask. Shortly after this study, the first RCT comparing HFNC with conventional oxygen devices after extubation was published [37]. The study included patients with acute respiratory failure due to pneumonia and trauma who were mechanically ventilated for a mean of almost five days before extubation. In these patients, the use of HFNC was associated with better comfort, better oxygenation, fewer desaturations and interface displacements, and a lower reintubation rate.

Postoperative patients remain an important subgroup of patients with some differences related to the response to HFNC. The effectiveness of HFNC therapy during the extubation period in postoperative patients remains controversial; most studies on this issue have included patients after cardiothoracic surgery. Parke et al. [38] included a non-selected population of cardiac surgery patients with mild to moderate acute respiratory failure, observing that HFNC patients more frequently succeeded and could be weaned to conventional oxygen devices. In contrast, in patients randomized to conventional oxygen devices, acute respiratory failure was more likely to worsen and escalation to NIV or HFNC required. Corley et al. [39] included a population of cardiac surgery patients with body mass index $(\mathrm{BMI}) \geq 30$ who were randomly assigned to prophylactic HFNC therapy or conventional oxygen devices after extubation. The authors did not observe any difference in atelectasis formation, oxygenation, respiratory rate, or dyspnea. Finally, the BiPOP study [28], a multicenter, non-inferiority RCT, compared HFNC and NIV for preventing or resolving acute respiratory failure after cardiothoracic surgery. Three different types of patient were eligible: patients who failed after a spontaneous breathing trial; patients who succeeded but had a preexisting risk factor for postoperative acute respiratory failure $(\mathrm{BMI}>30$, left ventricular ejection fraction $<40 \%$, and failure of previous extubation); and patients who succeeded after a spontaneous breathing trial but then failed extubation (defined as at least one of the following: $\mathrm{PaO}_{2} / \mathrm{FiO}_{2}<$
300 , respiratory rate $>25 \mathrm{bpm}$ for at least $2 \mathrm{~h}$, and use of accessory respiratory muscles or paradoxical respiration). After randomizing more than 800 patients, HFNC therapy did not increase the rate of treatment failure (defined as reintubation, switch to the other study treatment, or premature treatment discontinuation at the patient's request or due to an adverse event). Therefore, as HFNC therapy did not worsen outcomes, may be easier to administer, and requires lower nursing workload, the authors concluded that the results supported the use of HFNC in this subset of patients. Certain questions remain unanswered, however, such as the optimal flow and the subset of patients who would benefit the most from HFNC therapy.

Recently, Hernandez et al. [1] reported a multicenter randomized trial analyzing the effect of HFNC compared to conventional oxygen therapy in a population of lowrisk-of-reintubation patients. In the study, the authors aimed to evaluate whether high flow oxygen therapy after planned extubation would reduce the need for reintubation compared with standard oxygen therapy. The all-cause reintubation within $72 \mathrm{~h}$ was lower in the high flow group (4.9 vs $12.2 \%$ ). This difference was mainly attributable to a lower incidence of respiratory-related reintubations in the high flow group (1.5\% vs $8.7 \%)$. The main benefit was observed on reducing reintubation secondary to hypoxemia and inability to clear secretions. These results agree with those obtained by Maggiore et al. [37].

The authors classified patients according to the criteria for risk of reintubation. They did not use the type of respiratory failure considering that the aim was to obtain the preventive effects of HFNC and not treatment once the failure was present. It is currently unclear how to identify at risk patients for extubation failure. Previous trials, like that of Thille et al. [40], have tried to identify the underlying risks of extubation outcome. In keeping with these studies, this trial included 10 risk factors, which can clearly select a low risk population.

\section{High-risk-of-reintubation patients}

Simultaneously, Hernandez et al. [5] compared HFNC with NIV in patients at high risk for reintubation in a non-inferiority trial. While studies have suggested that prophylactic NIV could prevent postextubation respiratory failure, they appear inconsistent with regard to reintubation. However, Thille et al. [40] added new data supporting the benefit of NIV for this indication. In a general population of critical patients use of NIV has not been proved, for that reason in the low-risk group HFNC was compared to conventional oxygen therapy [1].

This study confirmed that the reintubation rate was non-inferior in the HFNC group compared to the NIV 
group within 72 h ( $22.8 \%$ vs $19.1 \%)$. For postextubation respiratory failure, the authors reported a lower rate in the HFNC group compared to the NIV group $(26.9 \%$ vs $39.8 \%$ ), suggesting that the postextubation respiratory failure rate could be even higher in the NIV group. This surprising result was explained by the significantly higher adverse event rate in the NIV group ( $43 \%$ vs $0 \%$ ), mainly discomfort and subsequent early withdrawal of the therapy (mean real time under NIV $14 \mathrm{~h}$, instead of the $24 \mathrm{~h}$ per protocol). This increased postextubation respiratory failure rate was not correlated with the reintubation rate, supporting a possible role of discomfort in NIV patients as the reason for the postextubation respiratory failure. In addition, the length of hospital stay was significantly reduced in the HFNC group [5].

\section{Facilitating weaning in tracheostomized patients}

Weaning of tracheostomy patients is still a challenge. To our knowledge, only one randomized trial has included high flow therapy in the protocol [41]. This was a singlecenter study including 181 critically ill tracheostomy patients who were randomized to have the tracheal cuff deflated or not during spontaneous breathing trials. All patients received high-flow conditioned oxygen therapy through a direct tracheostomy connection to the maximum tolerated flow and conditioned up to $37^{\circ} \mathrm{C}$. Although that study was not specifically designed to assess the effectiveness of high flow through the trachea, the authors hypothesized that HFNC therapy may have some benefits in the weaning process of tracheostomy patients with a deflated tracheal cuff. Positive airway pressure may theoretically reduce microaspiration and, with a deflated cuff, a higher flow is conveyed through the pericannular space, allowing for better drainage of secretions.

\section{Improving performance}

Regarding the methodology used in the protocol by Hernandez et al. [1], HFNC was applied before extubation to prevent the entrance of dry and cold air into the patient's native airway from the start of treatment. Although this is speculative, it could play a major role in the early benefit that was found in the lower rate of upper airway obstruction (laryngeal edema requiring reintubation was not observed in the HFNC group).

Flow was titrated according to patient tolerance; initially set at $10 \mathrm{l} / \mathrm{min}$ and titrated upward in $5 \mathrm{l} / \mathrm{min}$ steps until patients experienced discomfort. Twelve hours after extubation, a steady state is usually obtained. In that low-riskof-reintubation study, authors observed a tolerated main gas flow of $31 \mathrm{l} / \mathrm{min}$, a moderate flow as compared to the main flow tolerated in the high-risk-of-reintubation study (50 l/min), reinforcing the idea that under these conditions the flow tolerated is a marker of severity.
Another point of the protocol used by Hernandez et al. [1] deserves mention: patients who tolerated the spontaneous breathing trial were reconnected with the previous ventilator settings for rest and clinical evaluation of airway patency. Some preliminary studies suggest that spontaneous breathing trials could lead to mild respiratory muscle fatigue that could somehow influence extubation success. However, clinical evidence supporting this hypothesis is lacking.

After $24 \mathrm{~h}$, high-flow therapy was stopped and, if necessary, patients received conventional oxygen therapy. Maggiore et al. [37] reported better results using high-flow for $48 \mathrm{~h}$ after extubation, with some of the variables showing significant improvement after 24 continuous hours of application, suggesting that some time-dependent effects could lead to improved performance of this therapy.

With this information in mind, clinicians should counterbalance efficacy and safety. On the one hand, the longer the duration of HFNC application, the greater the clinical efficacy. On the other hand, the longer the duration of HFNC, the greater the probability of delaying escalation of respiratory support when HFNC fails. In fact, as suggested by Kang et al. [30], applying HFNC in patients with respiratory failure according to clinical response could lead to delayed intubation. This could be associated with a worse outcome, as has been shown with NIV. This may be possible because HFNC increases comfort, oxygenation and may disguise respiratory distress. The results by Hernandez et al. [1], reinforce this idea; under a fixed 24-hour protocol after extubation, the time to reintubation was not increased, whether compared to conventional oxygen therapy in the low-risk-of-reintubation group or NIV in the high-risk-of-reintubation group. A 24-h limit probably helped physicians appreciate undertreated respiratory distress at an early stage and not delay reintubation. Nevertheless, the results confirmed that $24 \mathrm{~h}$ was enough to reduce the rate of reintubation.

HFNC does not delay reintubation under those conditions. This result can be attributed to the preventive intention, the fixed duration and to the predefined reintubation criteria.

\section{Conclusion}

Delivery of heated and humidified oxygen at high flow rates through nasal cannulas is now widely used in adult patients. Its mechanisms of action and potential clinical benefits can help to improve the management of patients with acute respiratory failure or during the weaning phase. With the currently available evidence, several questions still remain unanswered; there is strong evidence for some clinical indications, but for other situations without that evidence decisions on HFNC treatment should be individualized in each particular situation and institution, taking into account 
resources, and local and personal experience with all respiratory support therapies. However, HFNC therapy is an innovative and powerful technique that is currently changing the management of patients with respiratory failure.

\section{Acknowledgements}

The authors highlight the contributions of the Spanish Multidisciplinary Group of High Flow Supportive therapy in adults (HiSpaFlow).

\section{Funding}

Publication charges were funded by Fisher\&Paykel.

\section{Availability of data and materials}

Not applicable.

\section{Authors' contributions}

All the authors made substantial contributions to the work and drafting or revising it critically for important intellectual content. All the authors gave their final approval to the version submitted for publication.

\section{Competing interests}

Fisher\&Paykel support a post doctoral fellow in Medical Research at the Hospital del Mar Institute. OR and GH have travel expenses and GH has personal fees covered by Fisher\&Paykel.

\section{Consent for publication}

Not applicable.

\section{Ethics approval and consent to participate}

Not applicable.

\section{Author details}

'Department of Critical Care Medicine, Virgen de la Salud University Hospital, Ave de Berber, 45005 Toledo, Spain. ${ }^{2}$ Department of Critical Care Medicine, Vall d'Hebron University Hospital, 08035 Barcelona, Spain. ${ }^{3}$ Instituto de Salud Carlos III, Ciber Enfermedades Respiratorias (Ciberes), Madrid, Spain.

\section{Published online: 21 March 2017}

\section{References}

1. Hernández G, Vaquero C, González P, et al. Effect of postextubation highflownasal cannula vs conventional oxygen therapy on reintubation in lowrisk patients. A randomized clinical trial. JAMA. 2016;315:1354-61.

2. Möller W, Celik G, Feng S, et al. Nasal high flow clears anatomical dead space in upper airway models. J Appl Physiol. 2015:118:1525-32.

3. Patel A, Nouraei SAR. Transnasal Humidified Rapid Insufflation Ventilatory Exchange (THRIVE): a physiological method of increasing apnoea time in patients with difficult airways. Anaesthesia. 2015;70:323-9.

4. Rudolf $B$, Hohenhorst W. Use of apneic oxygenation for the performance of pan-endoscopy. Otolaryngol Head Neck Surg. 2013;149:235-9.

5. Hernandez G, Vaquero C, Colinas L, et al. High flow conditioned oxygen therapy for prevention of reintubation in critically il patients at high risk for extubation failure: a multicenter randomised controlled trial. JAMA. 2016; 316:1565-74.

6. Parke RL, McGuinness SP. Pressures delivered by nasal high flow oxygen during all phases of the respiratory cycle. Respir Care. 2013:58:1621-4.

7. Parke RL, Bloch A, McGuinness SP. Effect of very-high-flow nasal therapy on airway pressure and end-expiratory lung impedance in healthy volunteers. Respir Care. 2015;60:1397-403.

8. Riera J, Perez P, Cortes J, et al. Effect of high-flow nasal cannula and body position on end-expiratory lung volume: a cohort study using electrical impedance tomography. Respir Care. 2013;58:589-96.

9. Roca O, Perez-Teran P, Masclans JR, et al. Patients with New York Heart Association class III heart failure may benefit with high flow nasal cannula supportive therapy: high flow nasal cannula in heart failure. J Crit Care. 2013;28:741-6.

10. Roca O, Riera J, Torres F, et al. High-flow oxygen therapy in acute respiratory failure. Respir Care. 2010;55:408-13.
11. Sztrymf B, Messika J, Mayot T, et al. Impact of high-flow nasal cannula oxygen therapy on intensive care unit patients with acute respiratory failure: a prospective observational study. J Crit Care. 2012;27:324e9-324e13.

12. Sztrymf B, Messika J, Bertrand F, et al. Beneficial effects of humidified high flow nasal oxygen in critical care patients: a prospective pilot study. Intensive Care Med. 2011:37:1780-6.

13. Frat JP, Brugiere B, Ragot $\mathrm{S}$, et al. Sequential application of oxygen therapy via high-flow nasal cannula and noninvasive ventilation in acute respiratory failure: an observational pilot study. Respir Care. 2015;60:170-8.

14. Schwabbauer N, Berg B, Blumenstock G, et al. Nasal high-flow oxygen therapy in patients with hypoxic respiratory failure: effect on functional and subjective respiratory parameters compared to conventional oxygen therapy and non-invasive ventilation (NIV). BMC Anesthesiol. 2014;14:66-73.

15. Rittayamai N, Tscheikuna J, Praphruetkit N, et al. Use of high-flow nasal cannula for acute dyspnea and hypoxemia in the emergency department. Respir Care. 2015;60:1377-82

16. Lenglet $H$, Sztrymf $B$, Leroy $C$, et al. Humidified high flow nasal oxygen during respiratory failure in the emergency department: feasibility and efficacy. Respir Care. 2012;57:1873-8.

17. Schlapbach LJ, Schaefer J, Brady AM, et al. High-flow nasal cannula (HFNC) support in interhospital transport of critically ill children. Intensive Care Med. 2014:40:592-9

18. Parke RL, McGuinness SP, Eccleston ML. A preliminary randomized controlled trial to assess effectiveness of nasal high-flow oxygen in intensive care patients. Respir Care. 2011;56:265-70.

19. Roca O, de Acilu MG, Caralt B, et al. Humidified high flow nasal cannula supportive therapy improves outcomes in lung transplant recipients readmitted to the intensive care unit because of acute respiratory failure. Transplantation. 2015:99:1092-8.

20. Frat JP, Thille AW, Mercat A, et al. High-flow oxygen through nasal cannula in acute hypoxemic respiratory failure. N Engl J Med. 2015:372:2185-96.

21. Patel BK, Wolfe KS, Pohlman AS, et al. Effect of noninvasive ventilation delivered by helmet vs face mask on the rate of endotracheal intubation in patients with acute respiratory distress syndrome: a randomized clinical trial. JAMA. 2016:315:2435-41.

22. Liu Q, Gao Y, Chen R, et al. Noninvasive ventilation with helmet versus control strategy in patients with acute respiratory failure: a systematic review and meta-analysis of controlled studies. Crit Care. 2016;20:265-79.

23. Frat JP, Ragot S, Girault C, et al. Effect of non-invasive oxygenation strategies in immunocompromised patients with severe acute respiratory failure: a post-hoc analysis of a randomised trial. Lancet Respir Med. 2016:4:646-52.

24. Carteaux G, Millán-Guilarte T, De Prost N, et al. Failure of noninvasive ventilation for de novo acute hypoxemic respiratory failure: role of tidal volume. Crit Care Med. 2016:44:282-90.

25. Coudroy R, Jamet A, Petua P, et al. High-flow nasal cannula oxygen therapy versus noninvasive ventilation in immunocompromised patients with acute respiratory failure: an observational cohort study. Ann Intensive Care. 2016;6: 45-56.

26. Maitra S, Som A, Bhattacharjee S, et al. Comparison of high-flow nasal oxygen therapy with conventional oxygen therapy and noninvasive ventilation in adult patients with acute hypoxemic respiratory failure: A meta-analysis and systematic review. J Crit Care. 2016;35:138-44.

27. Monro-Somerville T, Sim M, Ruddy J, et al. The effect of high-flow nasal cannula oxygen therapy on mortality and intubation rate in acute respiratory failure: a systematic review and meta-analysis. Crit Care Med. 2016. Sept 8. Epub ahead of print.

28. Stéphan $F$, Barrucand $B$, Petit $P$, et al. High-flow nasal oxygen vs noninvasive positive airway pressure in hypoxemic patients after cardiothoracic surgery. JAMA. 2015;313:2331-9.

29. Lemiale $V$, Mokart D, Mayaux J, et al. The effects of a 2-h trial of high-flow oxygen by nasal cannula versus Venturi mask in immunocompromised patients with hypoxemic acute respiratory failure: a multicenter randomized trial. Crit Care. 2015;19:380-8

30. Kang BJ, Koh Y, Lim CM, et al. Failure of high-flow nasal cannula therapy may delay intubation and increase mortality. Intensive Care Med. 2015;41: 623-32.

31. Rello J, Pérez M, Roca O, et al. High-flow nasal therapy in adults with severe acute respiratory infection: a cohort study in patients with 2009 influenza A H1N1v. J Crit Care. 2012;27:434-9. 
32. Roca O, Messika J, Caralt B, et al. Predicting success of high-flow nasal cannula in pneumonia patients with hypoxemic respiratory failure: The utility of the ROX index. J Crit Care. 2016;35:200-5.

33. Hernández G, Vaquero C, García S, et al. High flow conditioned oxygen therapy for prevention of reintubation in critically ill patients: a preliminary cohort study. Int J Crit Care Emerg Med. 2015;1:2-9.

34. Ranieri VM, Rubenfeld GD, Thompson BT, et al. Acute respiratory distress syndrome: the Berlin Definition. JAMA. 2012;307:2526-33.

35. Antonelli $\mathrm{M}$, Conti $\mathrm{G}$, Esquinas $\mathrm{A}$, et al. A multiple-center survey on the use in clinical practice of noninvasive ventilation as a first-line intervention for acute respiratory distress syndrome. Crit Care Med. 2007:35:18-25.

36. Tiruvoipati R, Lewis D, Haji K, et al. High-flow nasal oxygen vs high-flow face mask: a randomized crossover trial in extubated patients. J Crit Care. 2010; 25:463-8.

37. Maggiore SM, Idone FA, Vaschetto $R$, et al. Nasal highflow versus Venturi mask oxygen therapy after extubation. Effects on oxygenation, comfort, and clinical outcome. Am J Respir Crit Care Med. 2014;190:282-8.

38. Parke R, McGuinness $\mathrm{S}$, Dixon $\mathrm{R}$, et al. Open-label, phase II study of routine high-flow nasal oxygen therpy in cardiac surgical patients. Br J Anaesth. 2013;111:925-31.

39. Corley A, Bull T, Spooner AJ, et al. Direct extubation onto high-flow nasal cannulae post-cardiac surgery versus standard treatment in patients with a BMI >30: a randomized controlled trial. Intensive Care Med. 2015;41:887-94.

40. Thille AW, Boissier F, BenGhezala $H$, et al. Easily identified at risk patients for extubation failure may benefit from noninvasive ventilation: a prospective beforeafter study. Crit Care. 2016:20:48-56.

41. Hernandez G, Pedrosa A, Ortiz R, et al. The effects of increasing effective airway diameter on weaning from mechanical ventilation tracheostomized patients: a randomized controlled trial. Intensive Care Med. 2013;39:1063-70.

42. Mokart D, Geay C, Chow-Chine L, et al. High-flow oxygen therapy in cancer patients with acute respiratory failure. Intensive Care Med. 2015;41:2008-10.

43. Vourc'h M, Asfar P, Volteau C, et al. High-flow nasal cannula oxygen during endotracheal intubation in hypoxemic patients: a randomized controlled trial. Intensive Care Med. 2015;41:1538-48.

44. Rittayamai N, Tscheikuna J, Rujiwit P. High-flow nasal cannula versus conventional oxygen therapy after endotracheal extubation: a randomized crossover physiologic study. Respir Care. 2014:59:485-90.

45. Lucangelo U, Vassallo FG, Marras E, et al. High-flow nasal interface improves oxygenation in patients undergoing bronchoscopy. Crit Care Res Pract. 2012;212:506-12

46. Simon M, Braune $S$, Frings $D$, et al. High-flow nasal cannula oxygen versus non-invasive ventilation in patients with acute hypoxaemic respiratory failure undergoing flexible bronchoscopy. A prospective randmised trial. Crit Care. 2014;18:712-21.

47. Jones PG, Kimona S, Doran O, et al. Randomized controlled trial of humidified high-flow nasal oxygen for acute respiratory distress in the emergency department: the HOT-ER study. Respir Care. 2015;61:291-9. 